\title{
Pastoraltheologische Elemente zur Analyse christlicher Rundfunkarbeit in Lateinamerika
}

\author{
von Attilio Hartmann
}

In einem Kontinent wie Lateinamerika, dessen Rundfunksystem von kommerziellen, lokalen Sendern bestimmt wird, ist eine gründliche pastoraltheologische Auseinandersetzung mit der Rolle eines christlichen Senders besonders deswegen notwendig, weil gerade die einfache Bevölkerung im Sender immer noch das wichtigste Mittel moderner Massenkommunikation sicht und erfährt. Daß gerade in Lateinamerika solche Überlegungen nicht an der gesellschaftlichen und politischen Wirklichkeit vorbeigehen können, ist selbstverständlich ${ }^{1}$.

Im Folgenden wird versucht, thesenförmig einige Elemente einer pastoral-theologischen Analyse christlicher Rundfunkarbeit aufzulisten, die aus den konkreten Erfahrungen der Kirche in Brasilien entstanden sind, darüber hinaus aber Bedeutung haben dürften.

\section{Ausgangspunkt und Grundlage}

Theologischer Ausgangspunkt und Grundlage jeder kirchlichen Verkündigung vor allem im Rundfunk bildet der Johannesprolog:

„Im Anfang war der Logos ... das Wort. Das Wort war in Gott und das Wort war Gott. Alles, was besteht, ist durch das Wort gemacht worden. Das Wort war das Leben, das die Menschen erleuchtet. Die das Wort aufnahmen, empfingen die Macht, Kinder Gottes zu werden ..., ja, ebenfalls das Wort zu werden. Und das Wort wurde Mensch, wurde Geschichte und errichtete sein Zelt unter den Menschen. Wir alle haben aus der Fülle des Wortes empfangen, Gnade über Gnade.“

\section{Grundsätzliche Elemente}

2.1 Einen christlichen Rundfunk schaffen heißt, an einen menschgewordenen Gott glauben, der dreifach an den Rand gedrückt wurde:

- als Kind - die soziale Randexistenz (wie ein Sinnbild von Abhängigkeit und Bedürftigkeit)

— als Galiläer - die politische Randexistenz ( Kann denn von Nazareth aus Galiläa etwas Gutes kommen?")

- als Armer - die wirtschaftliche Randexistenz (ohne die „Segensgüter“ des Gottes Israels)

2.2 Ein christlicher Rundfunk soll ein Mittel sein, das verbreitet, anfacht, vervielfältigt ..., ein Resonanzkasten des Kampfes des Volkes, erleuchtet durch den Glauben an Jesus, den Befreier, geoffenbart in der Bibel, die den Menschen immer in die Mitte der

P. Attilio Hartmann sj ist Regionaler Kommunikationskoordinator der Brasilianischen Bischofskonferenz in Sao Paulo; er ist Sekretär der ökumenischen "Uniâo Cristâ Brasilera de Communicacâo Social“ (UCBC). 
Geschichte stellt als das Subjekt, das die derzeitige sozial-politisch-wirtschaftliche Wirklichkeit verwandelt, indem es eine neue Sozialordnung sucht, in welcher der Mensch sich immer mehr als Bruder des anderen versteht und fühlt, erhaben über alle Unterschiede der Rasse, des sozialen Standes, der Religion und sich hinwegsetzt über alle Grenzen von Volk oder Nation.

2.3 Ein christlicher Rundfunk muß der Allgemeinbildung dienen, wobei man unter Bildung zu verstehen hat, daß eine Gesprächsatmosphäre geschaffen wird, in der der zu Erziehende und der Erzieher brüderlich miteinander verkehren und als Ziel eine Gesellschaft vor Augen haben, in welcher die Beteiligung am politischen Leben, die Verantwortung für die Verwaltung in der Wirtschaft, die Teilhabe an den Gütern des Verbrauchs, der Kultur und des Glaubens ein neues System von Bezichungen im gemeinschaftlichen und sozialen Leben schaffen.

2.4 Als das wichtigste Massenmedium der Armen muß der Rundfunk in der Auswahl seiner Hörerschaft den am meisten Benachteiligten im sozialen Bereich den Vorzug geben. Eine christliche Rundfunkanstalt muß ein lebendiges Zeichen dieser Entscheidung für die unteren Volksschichten sein, nicht um aus ihnen einen Gegenstand zu machen, d. h. ein Publikum, dem man Nachrichten übermittelt, die speziell für es gedacht sind, sondern sie muß das Mikrophon öffnen, damit diese unteren Volksschichten den christlichen Rundfunk als ibr Kommunikationsmedium benutzen. Eine christliche Rundfunkpolitik macht keine Programme für das Volk, auch nicht mit dem Volk; das Volk selbst, das entsprechend organisiert und koordiniert ist, stellt seine Programme zusammen.

2.5 In dem Maße als eine Gemeinschaft spürt, daß der betreffende Rundfunksender ibr Kommunikationsmittel ist, löst sich auch das Problem des Unterhalts: das Volk zahlt für den Dienst, den der Sender ihm leistet. Wenn das Mikrophon offen bleibt für den Weg des Volkes, dann wird sich das Volk auch der Verpflichtung bewußt, die es auf sich nimmt.

2.6 Die Botschaft, die eine christliche Rundfunkanstalt bringt, soll keine Lehre sein, sondern eine Nachricht, die gute Nacbricbt. (Und die gute Nachricht ist, auch und vor allem, die Nachricht vom Menschen, der stirbt infolge des Kampfes für die Wahrheit, für die Gerechtigkeit: am Kreuz, am Altar, in irgendeiner Nacht, in irgendeiner Straße, in irgendeinem Land ...) Es sind ja Ereignisse der Erlösung und Befreiung, welche die christliche Rundfunksendung ausmachen. Man erinnere sich daran, daß die Bibel ein umfangreicher Bericht von Erlösungstaten ist.

2.7 Wir leben im Zeitalter des Bildes. Der große Fehler vieler christlicher Sprecher ist, daß sie den Rundfunk benutzen, um zu lehren und zu predigen. Diese Art bleibt immer zu ausschließlich, zu vertikal, zu wenig positiv. Sie läßt keine Wende zur besseren Gestaltung zu, keine Belebung der Sendungen. Und jede echte Nachrichtenübermittlung endet dort und in dem Augenblick, wo der Zwang beginnt. Im Bildzeitalter muß man Bilder bringen, keine Begriffe, keine Lehre. Von erlösenden Ereignissen erzählen und erlösende Bilder bringen.

2.8 Ein anderer Fehler, den der christliche Nachrichtenübermittler macht: die Eile, die man hat, um "die Welt zu retten“. Der Mangel an Geduld in der Geschichte bringt es mit sich, daß wichtige Zeitepochen, die in dem Prozeß der christlichen Befreiung oft unwiederbringlich sind, völlig vernichtet werden. Viele möchten selbst den Boden bereiten, das Saatgut aussuchen, möchten selbst pflanzen, begießen, möchten das Wachstum bewirken und ernten. Alles möchten sie selbst machen. Darin steckt viel 
persönliche Eitelkeit, die nicht dem Evangelium entstammt: „Einer ist, der pflanzt, ein anderer, der erntet."

2.9 Die Anklage ist ein wesentlicher Bestandteil der Glaubensverkündigung. Selbst wenn ich keine fertige Lösung habe, aus einer Situation der Ungerechtigkeit herauszukommen ..., selbst wenn ich keine letztgültige Anregung bieten kann für die Befreiung von der Unterdrückung, muß ich dennoch die Unterdrückung und den Unterdrücker brandmarken. Ich darf nicht schweigen nur deshalb, weil ich keine Lösung habe; meine Anklage wird eine Kettenreaktion hervorrufen, die die Gemeinschaft mit einbezieht und die die Befreiung entstehen läßt als Kampf und Sieg aller.

2.10 Einen christlichen Rundfunk schaffen heißt, einen politischen Vorschlag machen für den sozialen Wandel. Es bedeutet, daß man die Neutralität ablehnt, die ja gegen das Evangelium ist, und sich entscheidet für jenen Vorschlag, den Kampf des Volkes zu seinem eigenen Kampf macht. Als Strategie und Erziehung wird diese Entscheidung um so wirksamer sein, je weniger amtlich sie ist: das Amtliche übt Zwang aus, während das Nichtamtliche Anregungen gibt und Vorschläge macht...

2.11 Im Wirken des christlichen Rundfunks fehlt oft die friedfertige Aggressivität des Evangeliums. Es werden Programme ausgestrahlt, die den Eindruck des „gut Erzogenen“ machen, sie sind furchtsam; man betreibt eine Politik des „Bitteschön“, des „Wer weiß?", des „Wir schlagen vor, daß ...". Man muß die Lüge anklagen mit klaren und direkten Worten, ohne langes Herumreden: „Geht und sagt jenem Fuchs ... “, „ihr übertünchten Gräber“, „ihr Natterngezücht!“. Und man muß die Wahrheit verkünden, obwohl man sich genau der Gefahren bewußt ist, welche dies bedeuten kann. Hier müssen die christlichen Rundfunkmänner eines jeden Landes die Grenzen kennen, die ihnen gesetzt sind. Und sie dürfen den Kopf nicht verlieren ...

2.12 Vor allem darf man nicht die zarte und lyrische Seite der Angelegenheit aus dem Auge verlieren: "Wenn ihr nicht werdet wie ein Kind, werdet ihr nicht in das Reich Gottes kommen." „Schaut auf die Lilien des Feldes ..., seht die Vögel ..." Viele christliche Rundfunksprecher, die im Kampf für das Volk stehen, erliegen der Versuchung der Gewalttätigkeit, wenn sie die Situationen der Ungerechtigkeit zu spüren bekommen, und dann gibt es in ihrem Herzen kein Zartgefühl und keine Lyrik mehr. Auf Gewalt antworten sie ebenfalls mit Gewalt. Ihr Kampf richtet sich gegen das System, gegen die Reichen, gegen jene Christen, die sich aus allem heraushalten, gegen die fernstehenden Armen, gegen die institutionalisierte Kirche, weil sie nicht "alles verkauft und es den Armen gibt ...", gegen die Zivilgewalt, gegen die Militärmacht, gegen die Wirtschaftsmacht, gegen die religiöse Macht. Sie sind Menschen des Contra. Thre Worte sind immer bitter, sie offenbaren die Verbitterung ihres Herzens. Unsere Welt erlebt eine echte Ausschöpfung des Negativen, des „contra“. Es ist höchste Zeit, sich für eine Sache zu entscheiden und konstruktive Arbeit zu leisten. Wenn man das Böse anklagt, ohne das Gute zu verkünden, bringt dies schnell eine Ermüdung mit sich und hinterläßt den bitteren Nachgeschmadk der Niederlage ... „Wehe euch, ihr Reichen ...; wehe euch, ihr Satten ..., die ihr jetzt lacht ...“ (Anklage); doch gleichfalls ... „Selig seid ihr, ihr Armen ...; selig, die ihr jetzt hungert ..., die ihr weint. Freut euch, denn euer Lohn wird groß sein“ (Verkündigung des Positiven).

\section{Das System und der christliche Rundfunk}

3.1 Wenn man einen christlichen Rundfunk schaffen will, muß man ständig die „Theologie des Risiko“ leben. (Dom Alois Lorscheider sagte: „Wenn ich zu wählen 
habe zwischen dem Nichtstun aus Furcht vor der Möglichkeit zu fehlen und dem Tun, auch auf die Gefahr hin, nicht das Richtige zu treffen, ziehe ich das Risiko vor.") Das Risiko auf sich nehmen bedeutet, die Unsicherheit mit in Kauf nehmen; in Unsicherheit arbeiten heißt, mit Vertrauen arbeiten. Das Vertrauen steht im umgekehrten Verhältnis zur Sicherheit: für die totale Sicherheit gibt es kein Vertrauen. Jesus ruft zum Vertrauen auf, indem er in der Unsicherheit des Alltags lebt: „Die Füchse haben ihre Höhlen, die Vögel ihre Nester; doch der Menschensohn hat keinen Platz, wo er sein Haupt hinlegen könnte". "Ihr glaubt an Gott, glaubt auch an mich ...". "Mut, ich habe die Welt besiegt ${ }^{c}$.

3.2 Eines der größten Mißverständnisse, deren ein christlicher Rundfunksender sich schuldig machen kann, ist die Tatsache, daß man die Sendungen zu einem Pastoralobjekt macht in der Auffassung, man betreibe besser Glaubensverkündigung, wenn man eine größere Hörerschaft habe. Der Kampf um die Hörerschaft enthält ein Element, das gegen das Evangelium ist: er muß oft lügen, um innerhalb des Systems unserer Rundfunkanstalten, das ja auf Gewinn aus ist, mit den anderen Sendern konkurrieren zu können. Die „Versuchung der Zahlen" einer großen Hörerschaft ist tatsächlich eine Versuchung, der die christlichen Sender ausgesetzt sind. In der Art, wie sie die Dinge handhaben, zeigen sie, daß sie praktisch nicht glauben, daß „Maria ihren erstgeborenen Sohn zur Welt brachte und ihn in eine Krippe legte, weil in der Stadt für sie kein Platz war". Es gab kein großes Publikum, um Jesus bei seinem Eintritt in die Geschichte zu empfangen: nur die Mutter, Josef, einige Hirten, Männer des einfachen Volkes. Der Geburtsort war weder ein Palast noch die große Hauptstadt, ja nicht einmal das kleine Bethlehem. Die Geburtsstelle befindet sich am Rande ...., sogar außerhalb des kleinen Bethlehems, in einer ländlichen Umgebung, auf den Fluren ...

3.3 Ein anderes Mißverständnis ist, daß man eine „Philosophic des Schacherns“ betreibt, wobei zweifelhafte Situationen entstehen, die zu einem Zeichen werden, das gegen das Evangelium zeugt: „Eure Rede sei ein Ja, wenn ja; ein Nein, wenn man nein sagen muß. Alles andere kommt vom Teufel." Besonders dann, wenn es um die Notwendigkeit geht, für die kommerzielle Werbung Raum zu schaffen, geht man sparsam mit der Wahrheit um und läßt sie sogar ganz verschwinden. Pilatus wollte schachern: "Wen wollt ihr, daß ich freigebe: Barabbas oder Jesus?" Und der Unschuldige wurde verurteilt ...

3.4 Der offen zutage tretende oder versteckte Inhalt der Botschaft des Systems führt zum Gefühl der Ohnmacht: „Mögen Sie noch so viel tun ..., noch so viel kämpfen ..., Sie werden es nicht erreichen; doch haben Sie Vertrauen zu uns, denn wir vermögen es, wir wissen es, wir machen es". Der christliche Sender kann nicht in naiver Weise ein Werkzeug des Systems sein, ein Verstärker oder Einpauker des ideologischen Apparates, sondern er muß sich nach dem Wort Jesu orientieren: „Ihr selbst müßt ihnen zu essen geben ...“ „Er sandte sie zu zweien aus ... Und sogar die Dämonen gehorchten ihnen."

3.5 Die Sendungen des Systems sind so beschaffen, daß sie von oben nach unten gehen, sie sind nicht positiv, sie lassen keinen Raum frei für das Wort des Gesprächspartners. Die Sendungen der christlichen Rundfunkanstalt schaffen, wie der Meister, einen Raum für das Gespräch, wo alle gleichberechtigte Subjekte der Nachrichtenübermittlung sind: „Frau, gib mir zu trinken“. „Unsere Väter haben auf diesem Berg Gott angebetet, aber ihr Juden ... Wenn der Messias kommt, er wird uns alles das lehren." "Frau, ich bin es, der mit dir spricht"." 
3.6 Das System verfälscht und manipuliert konkrete Angaben und Informationen, um Angst hervorzurufen, Furcht, Unsicherheit. Die Zahl der Arbeitslosen wächst - „sei still, beschwer dich nicht über deinen Lohn, denn es gibt ja viele, die Arbeit suchen ..." - Die Gewalttätigkeit ist weit verbreitet, die „Mörderschwadronen “ werden angestachelt. Der christliche Sender ist die Vergegenwärtigung der Guten Nachricht: "Geht zu Johannes und sagt ihm: Blinde sehen, Lahme gehen ..."

3.7 Für das System gilt der Mensch nur etwas, wenn er produziert, er etwas besitzt; er wird nur nach seiner Leistungsfähigkeit bewertet, nach der Menge. Als Ideal gilt der "homo faber", der mechanisch seinen Beitrag leistet zur Herstellung der Staatsprodukte, der mit Freude die Auszeichnung annimmt, der sich immer auf die Seite der gegebenen Situation stellt und auf die Regierung schaut als auf den "Großen Vater", der ihm die Nahrung gibt, damit er überleben kann ... Der christliche Sender muß das Werkzeug sein, das den „homo ludens" wiederherstellt, der sich nicht zufriedengibt mit arbeiten, essen, zeugen, sondern der den Raum und die notwendigen Bedingungen fordert, um ein Leben in der Freude der Kinder Gottes führen zu können.

3.8 Wie in vielen anderen Unternehmungen, so merkt man auch im Bereich des Rundfunkwesens der Kirche eine gewisse Verschwendung und eine Uberbetonung der eigenen Kräfte. Jeder will seinen Sender haben und gestaltet die Rundfunkprogramme, wie er sie versteht und wünscht. Man kann hier das lateinische Sprichwort: „Quod abundat, non nocet" nicht geltend machen, denn die Fülle bedeutet in den meisten Fällen eine schlechte Qualität der Programme, Vermehrung der Unkosten, Konkurrenz (manchmal unredlich als ein contra-Zeugnis), und alles das ganz und gar im Sinne des kapitalistischen Systems, im Sinne der Gewinnvergötzung, der Hervorbringung von Mythen, der Schändung der Kommunikationsmittel.

3.9 Die Einrichtung eines christlichen Rundfunksenders darf nicht einfach das Ergebnis des persönlichen Geschmacks eines Bischofs, eines Provinzialoberen, eines Pfarrvikars sein. Das erste und wichtigste ist: eine Gruppe, die den Kampf des Volkes auf sich genommen, diskutiert über die Einrichtung des Senders und sorgt dafür, daß die ganze Gemeinschaft sich dafür verantwortlich fühlt als für ihr eigenes Kommunikationsmittel. Es erübrigt sich zu sagen, daß alle Angestellten dieser Rundfunkanstalt Männer und Frauen sein müssen, die den Kampf ihrer Gemeinde zu dem ihrigen gemacht haben: wichtiger als eine "schöne Stimme“ - Mythus des Systems - ist die Stimme von jemandem, der sich mit seinem eigenen Volk identifiziert.

3.10 Das System pflegt den Mythus des Letztgültigen: „Das ist das Letzte ..., das Beste ..., das Vollkommenste ..." Jesus stellt die Dynamik des Vorläufigen wieder her: "Jedem Tag genügt seine eigene Sorge. " Der christliche Sender darf sich in keiner Angelegenheit von dem Willen leiten lassen, das letzte Wort darüber zu haben oder das letzte Wort zu sein. Er muß davon überzeugt sein, daß der Tag der Nachrichtenübermittlung das Heute ist, das sich auf die Erfahrungen von Gestern gründet und sich vertrauensvoll in das Abenteuer des Morgen stiirzt. Und, wie jedes Abenteuer, hat auch das Abenteuer der Schaffung eines christlichen Rundfunks vieles an sich, was sich nicht voraussehen läßt, viele Unbekannten, viele Angstmomente, die an die Angst des Herrn Jesus erinnern: „Vater, wenn es möglich ist, nimm diesen Kelch von mir ..." „Mein Gott, mein Gott, warum hast du mich verlassen!?“

3.11 Das kapitalistische System, das aus Lateinamerika seinen großen Marktplatz des Konsums macht, vergötzt den Privatbesitz, aus dem es den Erlösungsmythus bildet. Der christliche Rundfunk macht diesen Mythus zunichte, indem er mutig verkündet, 
daß „jeder Privatbesitz mit der Sozialhypothek belastet ist"; der christliche Rundfunk behauptet, daß das christliche Ideal darin besteht, daß man ,in der Welt ist, ohne von der Welt zu sein", daß man vollkommen eingefügt ist in die Wirklichkeit der Welt und dennoch sich niemals von den Dingen dieser Welt versklaven läßt.

3.12 Das System sagt, die Messe sei lediglich „das Gedächtnis des Todes und der Auferstehung Jesu". Der christliche Sender muß der liturgischen Zelebration dienen, crlöst wie ein demokratischer Raum, der wichtige Stellen des Weges und Kampfes markiert für die Befreiung derer, die an Jesus Christus glauben; der Sender wird zum Resonanzkasten, der diesen Raum vergrößert und in derselben Feier die in der Kirche versammelte Gemeinde vereinigt mit der Gemeinschaft aller, die durch die Radiowellen mit ihr verbunden sind.

\section{Anmerkungen:}

${ }^{1}$ Den Thesen dieses Beitrages liegt ein Arbeitspapier zugrunde, welches für eine Unda-Veranstaltung in Quito im Sommer 1982 geschrieben wurde. Die Ubersetzung aus dem Portugiesischen besorgte P. Maximilian Kopitz svd.

\section{SUMMARY}

In Latin America with is commercial radio system, the Christian radio network has a special role to play also because radio, more than other means of mass communications, reaches the people at the grassroot. Here the Christian station has a special obligation in defending and liberating man from commerce and suppression. Starting from the prologue of St. John's Gospel, some basic considerations on Christian radio work are given, as well as considerations on the role of a Christian radio within the broadcasting system of Latin America.

\section{RÉSUME}

Dans le système radiophonique commercial en Amérique Latine, les activités chrétiennes dans ce domaine ont une signification spéciale en vue que la radio peut rejoindre toutes les classes de la population plus facile que les autres moyens de la communication. L'émetteur drétien a ici une obligation spéciale pour la défense et la libération de l'homme du commerce et de la soupression. En commençant avec le prologue de l'Evangile de St. Jean, quelques considérations élémentaires sont présentées à ce sujet comme à la situation de l'émetteur drétien dans le système radiophonique en Amérique Latine.

\section{RESUMEN}

En América Latina con su sistema de radios comerciales, las redes de emisoras christianas tienen un importante papel que jugar, especialmente porque la radio, aún más que otros medios de communicación de masas, llega al pueblo en su misma base. Por esto las emisoras cristianas tienen la especial obligación de defender y liberar al hombre del comercio y de su aniquilamiento. Comenzando con el prólogo del Evangelio de San Juan, el autor expresa algunas consideraciones básicas acerca del trabajo que debe realizar una radio cristiana y agrega algunas ideas sobre el papel de la radio cristiana en su relación con el sistema de radiodifusión existente en Latino América. 